\title{
EDITORIAL
}

\section{Große Ereignisse}

\author{
Andreas Klee $\cdot$ Wladimir Sgibnev
}

(C) Springer-Verlag Berlin Heidelberg 2014

Große Ereignisse - ungeachtet der sozialen Konstruktion dessen, was als großes Ereignis zu gelten habe - dienen gerne als Bezugspunkte für unser heutiges Handeln. Mag sein, dass noch nie jemand etwas aus der Geschichte gelernt hat und der Verweis auf Vergangenes nicht immer ein valides Argument ist. Dennoch bieten vergangene große Ereignisse immer wieder Gesprächsanlässe und damit Gelegenheiten, durch die diachrone Perspektive neue Einblicke zu aktuellen Fragen zu gewinnen. Vor allem sind es runde Jubiläen, die die notwendige Aufmerksamkeit erzeugen, damit Gesprächsanlässe gesellschaftliche (und hier raumplanerische) Relevanz erlangen. 2014 gedenken wir des vor einhundert Jahren begonnenen Ersten Weltkrieges. Vor fünfzig Jahren, 1964, begann mit dem Shinkansen die Ära von Hochgeschwindigkeitszügen. Fünfundzwanzig Jahre ist es her, dass der eiserne Vorhang fiel und die Simpsons zum ersten Mal ausgestrahlt wurden. Jedes Ereignis, das liegt in der Natur der Sache, jährt sich irgendwann und bietet schließlich im Format eines runden Jubiläums genügend Stoff für einen Aufhänger wie Wortmeldungen, Gedenkveranstaltungen und Sammelbände.

Eben aus dieser Perspektive ist es erfreulich, wenn historisch rückblickende Arbeiten sich keines runden Jubiläums

Dr. A. Klee $(\bowtie)$

Akademie für Raumforschung und Landesplanung - Leibniz-

Forum für Raumwissenschaften,

Hohenzollernstraße 11,

30161 Hannover, Deutschland

E-Mail: klee@arl-net.de

Dr. W. Sgibnev

Leibniz-Institut für Länderkunde,

Schongauerstraße 9,

04328 Leipzig, Deutschland

E-Mail: w_sgibnev@iffl-leipzig.de als Aufhänger bedienen - wie der Beitrag von Ingrid Krau zum Wandel des Berufsbilds von Stadt- und Raumplanern. Von der Eröffnung des ersten raumplanerischen Studiengangs in Deutschland 1968 bis heute hat sich, so der Tenor des Artikels, das Selbstverständnis der Berufsgruppe radikal gewandelt: ,... vom relativ autonomen Start gegenüber der etablierten Planung hin zur Akzeptanz sich selbst regulierender Märkte... und inkrementalistischem Prozessdenken". Außerdem befasst sich der Beitrag mit der Diskrepanz zwischen Stadtplanern und Raumplanern und den Herausforderungen im Hinblick auf Organisation und Selbstverständnis, vor welchen beide Berufsgruppen aktuell stehen.

Weitere zwei Artikel dieses Heftes gehen auf zentrale thematische Herausforderungen des Berufsstandes ein Energiewende und Demographie - und berücksichtigen dabei insbesondere methodische Aspekte. Nikolas Ludwig und Stephan Bosch präsentieren eine Methode zur Berechnung von Windenergie-Ertragspotenzialen auf Offenlandund Waldstandorten in Bayern. Die von den Autoren auf der Grundlage dieses Modells identifizierten alternativen Standorte sollen eine systematische und sozialökologisch verträgliche Ausweisung von Windenergiestandorten ermöglichen und damit zu einer ausgewogenen Raumentwicklung beitragen.

Der Beitrag von Gesa Matthes befasst sich mit der Quantifizierung von Reurbanisierungstendenzen in der Stadtregion Hamburg. Sie beleuchtet unterschiedliche Methoden zur Messung von Reurbanisierung und führt auf dieser Grundlage eigene Berechnungen für den Analyseraum durch. Die Daten lassen den Schluss zu, dass die Kernstadt Einwohner vorwiegend aus Städten von außerhalb der Region gewinnt und gleichzeitig die Wegzugstendenz sinkt. Ebenso hebt die Studie die Bedeutung älterer Menschen als „Träger der Reurbanisierung“ hervor. 
Im nächsten (englischsprachigen) Beitrag analysieren Michael Bentlage, Anne Wiese, Arno Brandt, Alain Thierstein und Frank Witlox Wissensnetzwerke im maritimen Wirtschaftssektor. Dieser ist durch eine besondere räumliche Konstellation und eine spezifische Verflechtung lokaler und globaler Wissensträger gekennzeichnet. Kopräsenz und Einbindung in Netzwerke entscheiden über Innovations- und Wettbewerbsfähigkeit.

Schließlich geht Anne Hennig der Frage nach, ob Wohnungen im Besitz von Finanzinvestoren ein höheres Mietpreisniveau haben. Man ist geneigt, diese Frage schnell mit Ja zu beantworten, denn zahlreiche Pressemeldungen der vergangenen Jahre lassen diesen Schluss zu. Anhand einer Querschnittsbetrachtung an drei Beispielstandorten in Dresden, Lübeck und Wuppertal kann die Autorin jedoch zeigen, dass für Wohnungen der Finanzinvestoren keine signifikant überdurchschnittlichen Niveaus feststellbar sind. Damit wird auch deren Einfluss auf den Wohnungsmarkt möglicherweise überschätzt. Allerdings - und das räumt die Autorin auch selbst ein - würden zusätzliche Längsschnittbetrachtungen wichtige Erkenntnisse darüber bringen, ob sich in den vergangenen Jahren große Mietanpassungen ergeben haben, was zu einer anderen Beurteilung des Wohnungsmarktgeschehens in den untersuchten Städten führen würde. Es bleibt also weiterer Forschungsbedarf zu Zielen und Effekten der Wohnpolitik. Wie eine solche konkret aussehen könnte, steht auf einem anderen Blatt - und damit schlagen wir wieder den Bogen zu den Eingangsüberlegungen: Ist ein großer Wurf sinnvoller oder eine Politik der kleinen Schritte? Dafür haben auch wir kein Patentrezept. Nur eine Warnung: Egal wie es am Ende ausgeht, für inkrementelle Schritte werden, im Gegensatz zu großen Ereignissen, keine runden Jubiläen gefeiert. 